\title{
Recombinant Skeleton Using Junction Points in Skeleton Based Images
}

\author{
Mrs. Komala Lakshmi \\ Assistant Professor, \\ Department of Computer Science, \\ Coimbaote-6, Tamil Nadu, India.
}

\author{
Dr.M.Punithavalli \\ Dean, School of Computer sciences, \\ Dr.SNS Rajalakshmi College of Arts and SNRSONS \\ COLLEGE, \\ Coimbatore, Tamil Nadu, India
}

\begin{abstract}
We perform the task of combining two skeleton images and to produce the recombinant skeleton. We propose the recombinant skeleton algorithm to produce the recombinant skeletons. The existing skeleton representation has been taken and the merge vertex detection algorithm was used before applying the recombinant skeleton algorithm. We can design and apply this recombinant skeleton in motion detection, image matching, tracking, panorama stitching, 3D modeling and object recognition. We can generate or manufacture the true real time object from the recombinant skeleton produced. The proposed method utilize local search algorithm for junction validation. Our frame work suggests the range of possibility in getting the recombinant skeleton. The boundary is essential for any transformation hence the bamboo skeleton algorithm is deployed for computing the boundary and for storing the skeleton together with the boundary. Thus our representation is skeleton with border or outline. From this new skeleton representation the proposed recombinant is achieved.
\end{abstract}

Keywords- Recombinant skeleton; bamboo skeleton; valance skeleton point (VSP); core skeleton point(CSP); junction skeleton points (JSP).

\section{INTRODUCTION}

In the field of computer vision, recognition of objects in the images is a main and important task which should be performed for understanding the scene. This task may be done using different features of the objects with respect to related application. Some of these features are shapes of objects, color, brightness, motion vector, etc [1]. Based on the application, a feature or a combination of them may be useful and may be applied. Shape of object is a rich and effective feature of the object which has a key role for recognition of objects.In this paper, we aim to reconstruct the objects using their skeleton shape representation [2]. Interest point detection is a recent terminology in computer vision that refers to the detection of interest points for subsequent processing. An interest point is a point in the image which in general can be characterized as follows: it has a clear, preferably mathematically well-founded, definition, it has a well-defined position in image space, the local image structure around the interest point is rich in terms of local information contents, such that the use of interest points simplify further processing in the vision system, it is stable under local and global perturbations in the image domain, including deformations as those arising from perspective transformations (sometimes reduced to affine transformations, scale changes, rotations and/or translations) as well as illumination/brightness variations, such that the interest points can be reliably computed with high degree of reproducibility, Optionally, the notion of interest point should include an attribute of scale, to make it possible to compute interest points from real-life images as well as under scale changes. By applying the selected interest point as junction point [3]. we can produce the recombinant skeleton that is a unique stable that satisfies the skeleton topology .

\section{BACKGROUD STUDY}

The skeleton [4] is important for object representation and recognition in different areas, such as image retrieval and computer graphics, character recognition, image processing, and the analysis of biomedical images .Skeleton-based representations are the abstraction of objects, which contain both shape features and topological structures of original objects. Many researchers have made great efforts to recognize the generic shape by matching skeleton structures represented by graphs or trees .Because of the skeleton's importance, many skeletonization algorithms[5] have been developed to represent and measure different shapes. We can distinguish skeleton points into various types as follows.

\section{A. Simple Points.}

A simple point [6][7][8] is an object point which can be removed without changing the topology of the object .

\section{B. Regular points.}

Regular points on a 1D that have exactly two neighbors.

C. End-points.

End-points of a curve that have exactly one neighbor

D. Joints.

The thinness property can be easily checked if the junction points are known in advance. Some skeletonization methods directly identify junction points [9]. If junction points are not known in advance, they have to be identified with another method.

\section{E. Generating Points}

Every Skelton Point is linked to the boundary points that are tangential to its maximal circle .These are called generating points. 


\section{F. Junction points.}

Junction points (where curves meet) which can have three or more neighbors. A junction point[10] should satisfy the following skeleton properties.

1) it should preserve the topological information of the original object

2) the position of the skeleton should be accurate

3) it should be stable under small deformations

4) it should contain the centers of maximal disks, which can be used for reconstruction of original object

5) it should be invariant under Euclidean transformations ,such as rotations and translations, and

6) it should represent significant visual parts of objects.

Also if we remove that junction point, then the resulting skeleton will not be a best skeletal extraction of the original image.[11]

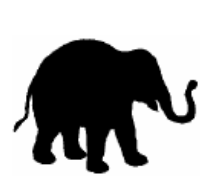

(a)

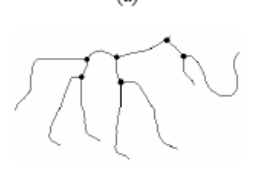

(c)

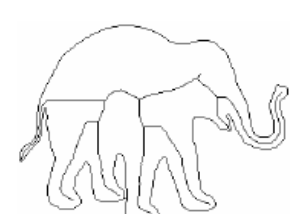

(b)

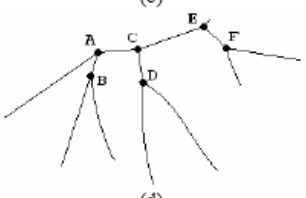

(d)
Fig2.1

(a): shape of an elephant, (b): boundary and skeleton, (c): skeleton with junction points, (d): connectivity graph of the skeleton

\section{Significance Of Juction Points}

Great progress has been made that define a significance measure for skeleton points and remove points whose significance is low. In [12] give a complete analysis and compare such pruning methods. Propagation velocity, maximal thickness, radius function, axis arc length, and the length of the boundary unfolded belong to the common significance measures of skeleton points. In [13] present a few significance measures for pruning complex Voronoi skeletons without disconnecting the skeletons. In [14] they combine a flux measurement with the thinning process to extract a robust and accurate connected skeleton.

\section{USAGE OF JUNCTION POINTS}

One of the biggest uses of skeletons is in analysis of scientific data where complex topologies can be easily explained using line-like drawings. Furthermore, skeletons can be used for reduced modeling and to explain simple physical phenomena. Examples include plume visualization [15], vortex core extraction [16], feature tracking [17] and many others. In all the above usage junction points play a vital role. Using these junction points it is possible to segment a digital image topologically into meaningful parts.This junction points play the vital role in the applications of skeletons namely computer vision, image analysis, and digital image processing, including optical character recognition, fingerprint recognition, visual inspection, pattern recognition, binary image compression, and protein folding [18].

\section{Methodology}

A. Step1.

Using bamboo skeleton algorithm to identify the boundary points.[19].

B. Step 2.

Using merge vertex detection algorithm to extract the merging point.[20].

C. Step3.

Deploying recombinant skeleton algorithm for merging the selected interest point (merge vertex derived from step 2) to obtain the junction point for the recombinant skeleton.

D. Step4.

Deploy the validation check for the skeleton properties and the junction point to confirm the recombinant skeleton produced

\section{RECOMBINANT SKELETON ALGORITHM}

1. For the first input image, compute the boundary points using bamboo skeleton algorithm

a) Bi $€$ IPI for all $i=1$ to $n$, $n$ is the set of all Pixels on the boundary.

b) Bj $€ I P 2$ for all $j=1$ to $m, m$ is the set of all pixels on the boundary.

2. For each Bi $€$ IP1 using merge vertex detection algorithm compute,

a) VSPi for all $i=1-n$

b) Fore each Bj $€ I P 2$ using merge vertex detection algorithm compute,

c) VSPj for all $j=1-m$

3. Using visual Data exploration

Let $r$ be the number of boundary points in the recombinant skeleton.

$$
\mathrm{rs}=\mathrm{m}+\mathrm{n}
$$

Compute the VSPi and VSPj combination to merge together so as to produce the junction points JSPx for $\mathrm{x}=1-\mathrm{rs}$.

Check for the validity of JSP using local search algorithm [21].

4. Confirm the JSP and connect the two images IP1 and IP2 to produce recombinant skeleton. The output recombinant skeleton produced is unique and stable and preserves the skeleton topology. 


\section{FLOW DIAGRAM}

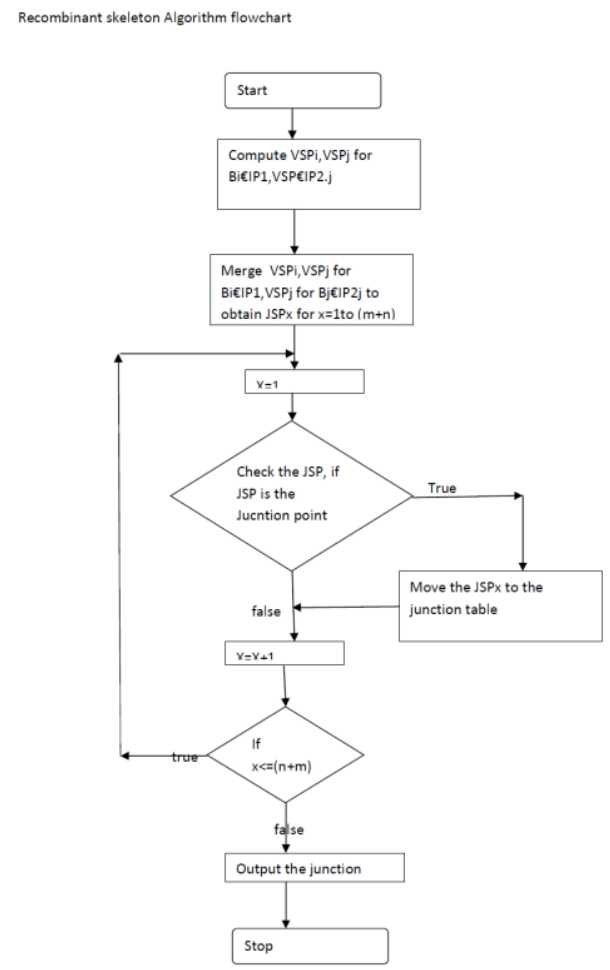

\section{RESULTS AND DISCUSSION}
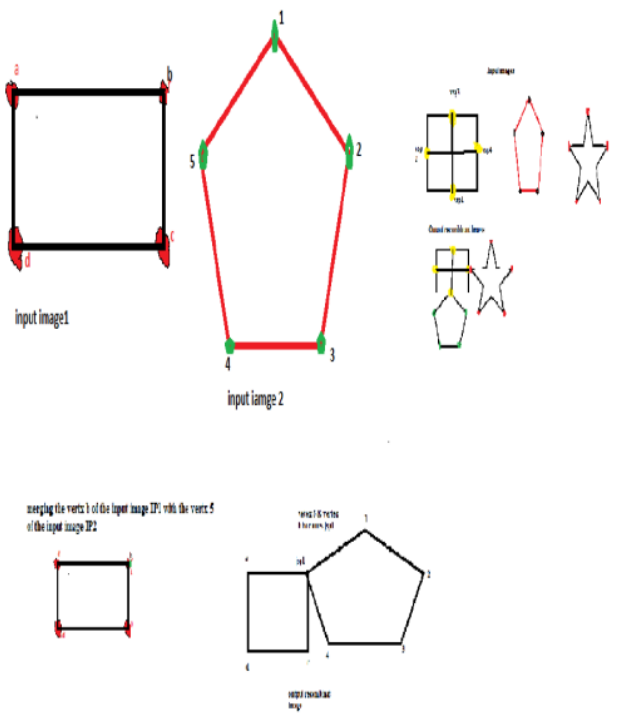

Fig 8.1

The main idea is to extract the boundary points [22] of the input image using bamboo skeleton algorithm. Say vsp1, vsp2,vsp3.vsp4 as in the fig 8.1.then compute the merging point [23] by testing the validity of the junction point and the stability of the skeleton and topological preservation.

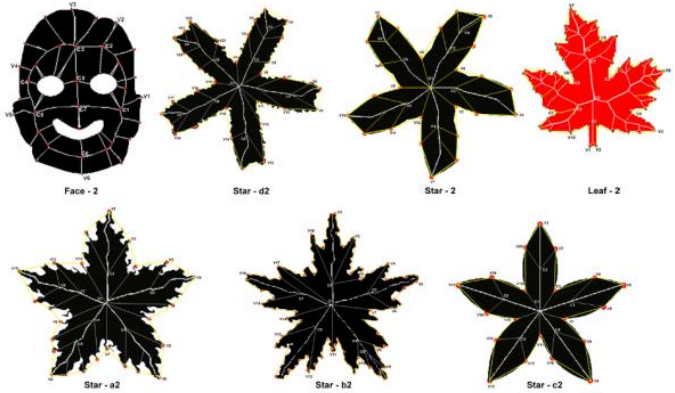

Fig 8.2
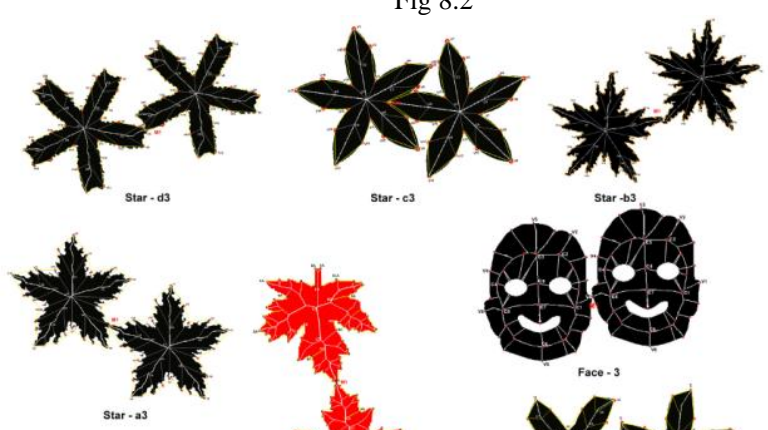

star-cas
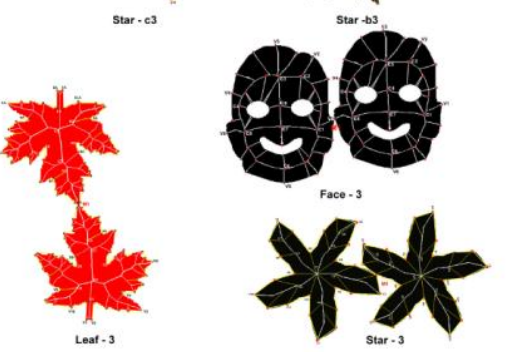

Fig 8.3

Combining the vertex points after the validity check and performance evaluation to obtain the recombinant skeleton structure. The results of the proposed method is depicted in fig 8.2, fig 8.3.

\section{A. . Contribution and Discussion}

We have presented an unconventional approach to shape representation and recognition by using skeletons.

1. The boundary point selected from the contour is independent of the threshold value. Whatever be the threshold value, the boundary points should not be removed and it is much more mandatory to remove the boundary deformation. In the existing discrete skeleton evolution method, the author utilizes the threshold value using global measure and hence there are Varity of different skeleton representation based on the threshold value[22], Where as in the proposed method, we do not remove the interested boundary point .

2. The boundary point selected, is checked for its positive maximum curvature .The boundary is formed with the pixels having positive maximum curvature value.[25]

3. Protrusion strength is used to check the interested skeleton point as the junction point.[26],to decompose the skeleton representation into meaningful parts. In this

Proposed method, when merging the two images, the protrusion strength of the merging point becomes the junction point of the recombinant shape representation. 
This point is having negative minima of curvature and the end point of that recombinant skeletal arc becomes the boundary point of the newly formed recombinant skeletal representation and the end point on that boundary should have the positive minima of curvature.

That is the two end points of the segment is of opposite extremum[27].

\section{Performance Evaluation}

As the bamboo skeleton algorithm is free from boundary deformation [24] and the mergevertx detection algorithm is unique and stable, the proposed recombinant skeleton algorithm [29] is also stable and preserves skeleton topology [28]. As the boundary is preserved, any shape matching algorithm can be used to retrieve the shape and recognize the objects.

\section{Conclusion And Future SCOPE}

The obtained skeleton is stable and unique and from that skeleton, we can produce or reconstruct object from skeleton that will definitely provides a platform for the research professionals to manufacture machinery parts from the skeletons of the various images in the databases. Soon we can produce galaxy man may be aliens to some other planets.

As the skeleton representation is derived from the input image by using digital image processing techniques, instead of moving to the recognition process, we are directed to reconstruct the given input image or new $2 \mathrm{D}$ objects from the recombinant skeleton representation.

Any modifications developed in the generated real time objects can also be studied and accordingly the work can be extended from objects point of view and its representation. The proposed tracking algorithms can be used for transforming the input image into the modified output image that could be used for further image processing. Also, it is possible to reconstruct the real time objects from the digital representation of the proposed method. The experimental results prove that it could be possible and achievable.

\section{ACKNOWLEDGMENT}

We thank X. Bai and L. Latecki for the skeleton and curvature code, and we also thank the authors of [22]. The author would like to express her gratitude to Mr. Dr .V. Sengoden, Principal and secretary, SNR SONS COLLEGE, COIMBATORE for providing necessary infrastructure and for his constant encouragement that led to improvise the presentation of the quality of the paper.

\section{REFERENCES}

[1] H. Blum. A Transformation for Extraction New Descriptors of Shape, Models for the Perception of Speech and Visual Form, MIT Press, 1967.

[2] T.Y. Kong, A. Rosenfeld. Digital topology: Introduction and survey. Comp. Vision, Graphics, and Image Proc., 48(3):357-393, 1989.

[3] Mrs. J.KomalaLakshmi,and Dr.M.Punithavalli,"A Survey on junction points in digital Image Processing" Paper accepted for the international conference IACC'09 IEEE Delhi Section Thapar University, Patiala147004 Punjab India
[4] J.Komala Lakshmi and M.Punitha Valli:A Survey on skeletons in digital image processing, in the international conference proceedings of IEEE Computer Society, 2009:260-269.

[5] J.Komala Lakshmi and M.Punitha Valli:A Survey on skeletonization in digital image processing, in the international conference proceedings of Managing Next Generations Software Applications08,Sponsored by CSIR NewDelhi,2008:825-839

[6] G. Bertrand and Z. Aktouf. A three-dimensional thinning algorithm using subfields, Vision Geometry III, 2356:113-124. SPIE, 1994.

[7] T.Y. Kong, A. Rosenfeld. Digital topology: Introduction and survey. Comp. Vision, Graphics, and Image Proc., 48(3):357-393, 1989.

[8] T.Y. Kong, A.W. Roscoe, A. Rosenfeld. Concepts of digital topology, Topology and its App., 46(3):219-262, Elsevier, 1992.

[9] J. Chuang, C. Tsai, Min-Chi Ko. Skeletonization of Three-Dimensional Object Using Generalized Potential Field, IEEE PAMI, 22(11):12411251,2000

[10] Detection and characterization of junctions in a 2D image Computer Vision and Image Understanding, Volume 93, Issue 3, March 2004, Pages 288-309

[11] F.F. Leymarie. 3D Shape Representation via Shock Flows, Ph.D. thesis, Brown University, May 2003.

[12]. D. Shaken and A. M. Bruckstein. Pruning Medial Axes. Computer Vision and Image Understanding,69(2): 156-169, 1998.

[13]. R. L. Ogniewicz, O. Kübler, Hierarchic Voronoi skeletons, Pattern Recognition, 28 (3): 343 -359,1995.

[14]. K. Siddiqi, S. Bouix, A. R. Tannenbaum, S. W. Zucker. HamiltonJacobi Skeletons. International Journal of Computer Vision, 48(3): 215231, 2002.

[15] K. Santilli, K. Bemis, D. Silver, J. Dastur, P. Rona. Generating realistic images from hydrothermal plume data, IEEE Vis., 2004.

[16] D.C. Banks, B.A. Singer. Vortex tubes in turbulent flows: identification, representation, reconstruction, IEEE Vis., 1994.

[17] B. Vrolijk, F. Reinders, F.H. Post. Feature tracking with skeleton graphs, in Data Visualization: The State of the Art, pp. 37--52, Kluwer Academic Publishers, 2003.

[18] A Transformation for Extracting New Descriptors of Shape" by H. Blum, in Models for the Perception of Speech and Visual Form, W. WhatenDunn (Ed.). MIT Press: Cambridge, MA, pp. 362-380R. Bergevin and A. Bubel

[19] J.Komala Lakshmi and M.Punitha Valli,"discrete skeleton reconstruction using bamboo skeleton"communitcated to IEEE transaction on image processing.

[20] J.Komala Lakshmi and M.PunithaValli "Impact of Boundary points in skeleton based images " in the international journal of advanced engineering and applications IJAEA: An International Journal of Advanced Engineering \& Applications Year 2010, Volume-1, June Issue,Print ISSN : 0975 - 7783,Online ISSN : 0975 - 7791,_stepsindia.com/ijaea/cfp.html

[21]Shape Recognition by Clustering and Matching of Skeletons Hamidreza Zaboli Amirkabir University of Technology, Tehran, Iran

[22]. Xiang Bai and Longin Jan Latecki, "Discrete Skeleton Evolution", EMMCVPR,Ezhou, China, 2007. (Applications: visual skeletons, corner detection, shape decompostion)

[23] J.Komala Lakshmi and M.Punitha Valli," Published an article titled ,Computation of merging points in skeleton based digital images" in the Global Journal Of computer science and technology GJCST Volume 9Number 4 September 2009 Issue._www.globaljournals.org Print ISSN : 0975 - 4350, Online ISSN : 0975 - 4172

[24] Xiang Bai, Longin Jan Latecki, and Wen-Yu Liu: "Skeleton Pruning by Contour partitioning with Discrete Curve Evolution," IEEE Trans. PAMI, 29(3): 449-462, 2007

[25] Aslan, C.; Erdem, A.; Erdem, E.; Tari, S.; Microsoft Corp., Redmond, WA ,Disconnected Skeleton: Shape at Its Absolute Scale, pattern analysis and machine intelligence, ieee transaction on,IEEE computer society,Volume: 30 Issue :12, 02 February 2008 
[26] JingTing Zeng, Rolf Lakaemper, XingWei Yang, Xin Li,Temple University, Philadelphia, PA. 2D Shape Decomposition Based on Combined Skeleton-Boundary Features, Lecture Notes in Computer Science

[27]Michael Leyton, Symmetry-Curvature Duality, computer vision ,graphics and image processing,38, 327-341 (1987).

[28] J. komala lakshmi and M.punithavalli, Published an article titled "2D shape reconstruction based on combined skeleton boundary features" in the international journal of image processing,

ISSN (Online) 1985-2304,Volume 4 - Issue 4 September 2010, Malaysia,_www.cscjournals.org

[29] J. komala lakshmi and M.punithavalli, "a survey on Performance evaluation in object detection techniques of Digital Image Processing " Internation journal of Computer science Issues vol 7,Issue 6,November 2010.ISSN Online 1694-0814,IJCSI,Maritius.

\section{AUTHORS PROFILE}

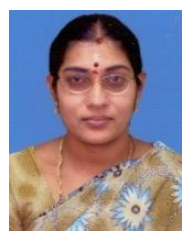

The author Mrs J.Komala Lakshmi, has finished her B.Sc Mathematics from SeethaLakshmi Achi College for women ,Pallathur, during 1995.She has completed her M.C.A from J.J.college Of arts and Sciences, Pudhukottai during 1998. She has been Awarded her M.Phil Computer Science,Bharathiar University during 2008. She Has been Awarded with the Silver meadal for her academic Excellence in M.C.A from the Institution from. She has Nine years of teaching experience in Collegiate service. She is currently working as Assistant Professor, Department of Computer Science, SNR SONS COLLEGE, Coimbatore. She has presented her papers in three international conferences. She has published three papers in four international journals. She also contributed a book publications to the computer science students. She was a Graduate Student Member of IEEE. She has been awarded with the recognition from Who's Who in the World 2011, 28th Edition for her notable contribution to the society. 